\title{
Transition State Theory For Laser-Driven Reactions
}

Shinnosuke Kawai

André D. Bandrauk

Charles Jaffé

Thomas Bartsch

Jesús Palacián

See next page for additional authors

Follow this and additional works at: https://researchrepository.wvu.edu/faculty_publications

\section{Digital Commons Citation}

Kawai, Shinnosuke; Bandrauk, André D.; Jaffé, Charles; Bartsch, Thomas; Palacián, Jesús; and Uzer, T., "Transition State Theory For Laser-Driven Reactions" (2007). Faculty Scholarship. 712.

https://researchrepository.wvu.edu/faculty_publications/712 
Authors

Shinnosuke Kawai, André D. Bandrauk, Charles Jaffé, Thomas Bartsch, Jesús Palacián, and T. Uzer 


\section{Transition state theory for laser-driven reactions}

Shinnosuke Kawai, André D. Bandrauk, Charles Jaffé, Thomas Bartsch, Jesús Palacián, and T. Uzer

Citation: The Journal of Chemical Physics 126, 164306 (2007); doi: 10.1063/1.2720841

View online: https://doi.org/10.1063/1.2720841

View Table of Contents: http://aip.scitation.org/toc/jcp/126/16

Published by the American Institute of Physics

\section{Articles you may be interested in}

Transition state theory for activated systems with driven anharmonic barriers

The Journal of Chemical Physics 147, 074104 (2017); 10.1063/1.4997571

Chemical reactions induced by oscillating external fields in weak thermal environments

The Journal of Chemical Physics 142, 074108 (2015); 10.1063/1.4907590

Communication: Transition state trajectory stability determines barrier crossing rates in chemical reactions induced by time-dependent oscillating fields

The Journal of Chemical Physics 141, 041106 (2014); 10.1063/1.4891471

Identifying reactive trajectories using a moving transition state

The Journal of Chemical Physics 124, 244310 (2006); 10.1063/1.2206587

Reaction rate calculation with time-dependent invariant manifolds

The Journal of Chemical Physics 136, 224510 (2012); 10.1063/1.4726125

Quantum reaction boundary to mediate reactions in laser fields

The Journal of Chemical Physics 134, 024317 (2011); 10.1063/1.3528937

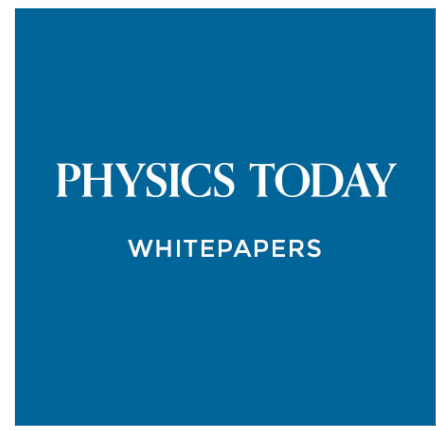

ADVANCED LIGHT CURE ADHESIVES

Take a closer look at what these environmentally friendly adhesive systems can do

\section{READ NOW}

PRESENTED BY

8. MASTERBOND 


\title{
Transition state theory for laser-driven reactions
}

\author{
Shinnosuke Kawai ${ }^{\mathrm{a})}$ and André D. Bandrauk \\ Laboratoire de Chimie Théorique, Faculté des Sciences, Université de Sherbrooke, Sherbrooke, \\ Québec J1K 2R1, Canada \\ Charles Jaffé \\ Department of Chemistry, West Virginia University, Morgantown, West Virginia 26506-6045 \\ Thomas Bartsch \\ Department of Mathematical Sciences, Loughborough University, Loughborough LE11 3TU, \\ United Kingdom \\ Jesús Palacián \\ Departamento de Ingeniería Matemática e Informática, Universidad Pública de Navarra, \\ 31006 Pamplona, Spain \\ T. Uzer \\ Center for Nonlinear Sciences, School of Physics, Georgia Institute of Technology, Atlanta, \\ Georgia 30332-0430
}

(Received 23 January 2007; accepted 6 March 2007; published online 24 April 2007)

Recent developments in transition state theory brought about by dynamical systems theory are extended to time-dependent systems such as laser-driven reactions. Using time-dependent normal form theory, the authors construct a reaction coordinate with regular dynamics inside the transition region. The conservation of the associated action enables one to extract time-dependent invariant manifolds that act as separatrices between reactive and nonreactive trajectories and thus make it possible to predict the ultimate fate of a trajectory. They illustrate the power of our approach on a driven Hénon-Heiles system, which serves as a simple example of a reactive system with several open channels. The present generalization of transition state theory to driven systems will allow one to study processes such as the control of chemical reactions through laser pulses. (C) 2007 American Institute of Physics. [DOI: 10.1063/1.2720841]

\section{INTRODUCTION}

The transition state (TS), a dividing hypersurface between the reactant and the product regions in phase space, ${ }^{1-3}$ is one of the central concepts in the theory of chemical reactions. It plays an important role in determining the rate constant and also provides an intuitive understanding of the reaction through the geometrical structure of the phase space. The validity of TS theory is based on the "no-recrossing" assumption, which demands that all the reactive trajectories that go from the reactant to the product region or vice versa must cross the TS once and only once, whereas nonreactive trajectories do not cross it at all. Through a recent approach to TS theory ${ }^{1-9}$ based on the geometric theory of dynamical systems, a dividing surface that satisfies this condition can be constructed for autonomous Hamiltonian systems with arbitrarily many degrees of freedom. The approach assumes only that the reactant and product regions are separated by an energy barrier, i.e., a rank-1 saddle point of the effective potential, where the local dynamics decouples into a single unstable reactive mode and several stable bath modes. The dividing surface thus obtained is bounded by a normally hyperbolic invariant manifold (NHIM). ${ }^{10}$ The stable and unstable manifolds of the NHIM act as separatrices between

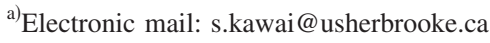

reactive and nonreactive trajectories that funnel enclosed trajectories towards and away from the TS. (An analogous mechanism had previously been described in system with two degrees of freedom. ${ }^{11-13}$ ) These manifolds encode a detailed microscopic description of the reaction dynamics.

On a different front, the development of laser technology ${ }^{14-17}$ in the past decades has led to laser pulses whose duration is on the time scale of the molecular motion, that is, pico- or femtosecond. It is thus becoming feasible to manipulate and control chemical reactions on a microscopic level through the application of judiciously shaped laser pulses (see Refs. 14, 15, and 18 for reviews). However, to determine the required pulse shapes one needs to understand the dynamics of laser-driven reactions in microscopic detail. The geometric TS theory has the potential to provide such knowledge if it can be generalized to problems with external driving fields. This is the aim of the present paper.

Recent work by Bartsch et al. ${ }^{19-21}$ on TST for timedependent problems has addressed the inclusion of stochastic time-dependent forces (due to solvents in liquid phase reactions, for example). Starting from the Langevin equation of motion, they showed the existence of a "transition state trajectory," which stays in the vicinity of the barrier for all time. The TS trajectory generalizes the saddle point that is of central importance to autonomous TST to the time-dependent 


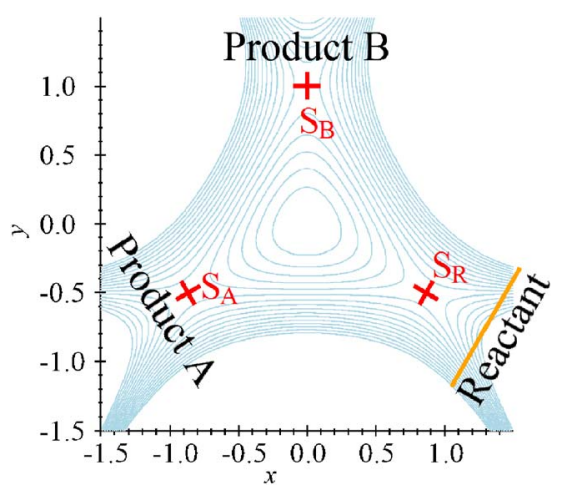

FIG. 1. (Color online) Contour plot of the potential energy of Hénon-Heiles system. Contours are spaced by 0.03 . The saddle points $S_{A}, S_{B}$, and $S_{R}$ separate a central region from three different asymptotic channels. The line in the reactant channel indicates the dividing surface used to sample initial conditions.

setting. Time-dependent invariant manifolds that separate reactive from nonreactive trajectories in the known manner are attached to the TS trajectory.

In this paper, we combine the concept of the TS trajectory with the method of normal form (NF) expansions based on Lie transformations ${ }^{22}$ which has been shown to be an effective tool to calculate invariant manifolds in autonomous systems $^{1-9}$ (see also Refs. 23 and 24 for a quantum version of Lie transformations). Preceding work on time-dependent normal form (TDNF) in the field of celestial mechanics ${ }^{25}$ assumes the external driving to be periodic or quasiperiodic, which precludes an application of the algorithm to short laser pulses. In contrast, the TDNF scheme developed here can be applied to any form of time dependence of the external force. We will compare the result of the TDNF with those of a harmonic approximation and of time-independent NF theory and thereby demonstrate that both the nonlinearity and the time dependence are equally important and can be correctly handled by TDNF theory.

Section II presents the technical details of the TDNF scheme. The basic finding there is that, although resonances between the external field and the bath modes do not allow reducing the dynamics to an integrable $\mathrm{NF}$, it is possible to separate the reactive mode from all other modes even under the influence of the laser field. This separation yields a constant of the motion, viz., the action variable associated with the reactive mode. The sign of this action variable distinguishes reactive from nonreactive trajectories and thus also characterizes the separatrices between them.

In Sec. III, an application of the TDNF to a simple twodegree of freedom model is given: a Hénon-Heiles potential with a Gaussian interaction with the external field. That potential, ${ }^{26}$ whose contour plot is shown in Fig. 1, has one minimum at the origin and three saddle points that separate a central region from three asymptotic regions. We regard the asymptotic regions as depicting the reactant channel and two different product channels, the central region as corresponding to an intermediate activated complex. The system then serves as a simple model for multichannel reactions such as

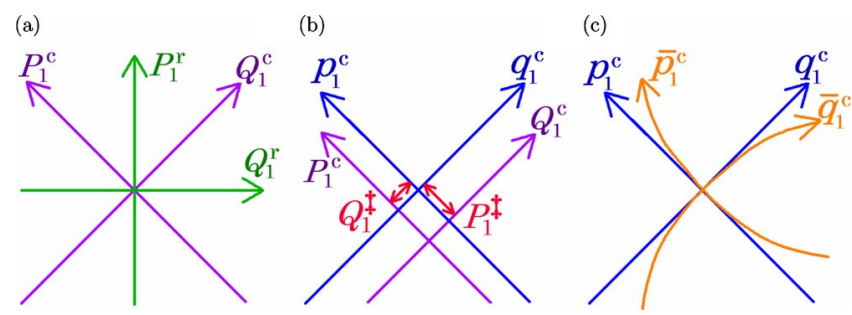

FIG. 2. (Color online) The main steps of the time-dependent normal form method (schematic). (a) Diagonalization of the linearized autonomous dynamics, $\left(\boldsymbol{Q}^{r}, \boldsymbol{P}^{r}\right) \mapsto\left(\boldsymbol{Q}^{c}, \boldsymbol{P}^{c}\right)$. (b) Time-dependent shift of the origin to the transition state trajectory $\left(\boldsymbol{Q}^{c}, \boldsymbol{P}^{c}\right) \mapsto\left(\boldsymbol{q}^{c}, \boldsymbol{p}^{c}\right)$. (c) Nonlinear normal form transformation $\left(\boldsymbol{q}^{c}, \boldsymbol{p}^{c}\right) \mapsto\left(\overline{\boldsymbol{q}}^{c}, \overline{\boldsymbol{p}}^{c}\right)$.

$$
R_{1}+R_{2} \leftrightharpoons M^{*}\left\{\begin{array}{l}
\rightarrow P_{1}+P_{2} \\
\rightarrow P_{1}^{\prime}+P_{2}^{\prime},
\end{array}\right.
$$

where $R_{1}$ and $R_{2}$ denote reactant molecules that collide to form a metastable complex $M^{*}$, which then dissociates either into one of the two product channels $P_{1}+P_{2}$ or $P_{1}^{\prime}+P_{2}^{\prime}$, or back into the reactant channel $R_{1}+R_{2}$.

The laser field influences both the formation and the decay of the intermediate complex. Some of the trajectories starting in the reactant region of phase space are simply reflected by the first barrier (marked by $S_{R}$ in Fig. 1) and never form the complex, whereas others surmount the barrier and reach the intermediate region. Once the complex has been formed, it can decay across any of the three barriers $\left(S_{R}, S_{A}, S_{B}\right)$ that lead into the three channels. The outcome of the reaction is determined by the channel chosen. It is therefore important to specify the conditions for a trajectory to enter into the intermediate region in the first place and then to react into a given channel. We will construct timedependent normal forms around all three saddle points and show that the invariant manifolds extracted in this way provide such conditions that allow one to predict the ultimate fate of a trajectory a priori, without having to carry out a numerical simulation.

\section{THEORY}

In this section, we develop a scheme to calculate the phase space structures in the vicinity of a saddle point for laser-driven reactions. The algorithm, which is based on time-dependent normal form theory, consists of three main steps that are illustrated schematically in Fig. 2: (a) diagonalization of the linearized time-independent dynamics, (b) a time-dependent shift of origin that eliminates the time dependence to lowest order, and (c) a sequence of nonlinear coordinate transformations that reduces the dynamics to a suitably chosen normal form. As a result, we will identify a reactive mode that can be separated from all other modes even under the influence of the external field. Although resonances between the bath modes and the external field prevent the reduction of the Hamiltonian to an integrable normal form, it is possible to construct one constant of the motion, viz., the action variable of the reactive mode. It defines phase space structures such as separatrices between reactive and nonreactive trajectories.

We start with a general Hamiltonian of the form 


$$
H^{\mathrm{tot}}(\boldsymbol{q}, \boldsymbol{p}, t)=H^{\mathrm{sys}}(\boldsymbol{q}, \boldsymbol{p})+H^{\mathrm{ex}}(\boldsymbol{q}, \boldsymbol{p}, t),
$$

where $H^{\text {sys }}$ is the Hamiltonian of the isolated system, and $H^{\text {ex }}$ describes the interaction with the time-dependent external field.

We assume that $\boldsymbol{q}=\boldsymbol{p}=0$ is a rank- 1 saddle point of $H^{\mathrm{sys}}$, i.e., it denotes the location of an energy barrier. We expand the Hamiltonian in Taylor series as follows:

$$
\begin{aligned}
& H^{\mathrm{sys}}(\boldsymbol{q}, \boldsymbol{p})=\sum_{\nu=2}^{\infty} \sum_{\Sigma_{\ell}\left(j_{\ell}+k_{\ell}\right)=\nu} \alpha_{j k} q_{1}^{j_{1}} \cdots q_{n}^{j_{n}} p_{1}^{k_{1}} \cdots p_{n}^{k_{n}}, \\
& H^{\mathrm{ex}}(\boldsymbol{q}, \boldsymbol{p}, t)=\sum_{\nu=1}^{\infty} \sum_{\Sigma_{\ell}\left(j_{\ell}+k_{\ell}\right)=\nu} \beta_{j k}(t) q_{1}^{j_{1}} \cdots q_{n}^{j_{n}} p_{1}^{k_{1}} \cdots p_{n}^{k_{n}},
\end{aligned}
$$

where the exponents $j_{\ell}$ and $k_{\ell}(\ell=1, \ldots, n)$ are non-negative integers and $\alpha_{j k}$ and $\beta_{j k}(t)$ are expansion coefficients. The expansion of $H^{\text {sys }}$ begins with $\nu=2$ because $\boldsymbol{q}=\boldsymbol{p}=0$ is an equilibrium point. The expansion of $H^{\mathrm{ex}}$ starts with $\nu=1$ since terms with $\nu=0$ have no influence on the motion and can therefore be neglected. For example, a dipole interaction with an external electric field leads to $\beta_{j k}(t)=0$ for $\boldsymbol{k} \neq \mathbf{0}$ and $\beta_{j 0}(t)=\mathcal{E}(t) \cdot \boldsymbol{\mu}_{j}$, where $\mathcal{E}(t)$ is the electric field and

$$
\boldsymbol{\mu}(\boldsymbol{q})=\sum_{\nu=1}^{\infty} \sum_{\Sigma_{\ell j_{\ell}=\nu}} \boldsymbol{\mu}_{j} q_{1}^{j_{1}} \cdots q_{n}^{j_{n}}
$$

is the dipole moment of the system as a function of the nuclear coordinates $\boldsymbol{q}$.

Since the NF theory is a perturbative approach, we introduce formally a perturbation parameter $\varepsilon$ that will be set equal to 1 in the end. We scale according to

$$
\boldsymbol{q} \mapsto \varepsilon \boldsymbol{q}, \quad \boldsymbol{p} \mapsto \varepsilon \boldsymbol{p}, \quad \beta_{j k}(t) \mapsto \varepsilon \beta_{j k}(t), \quad H^{\mathrm{tot}} \mapsto \varepsilon^{-2} H^{\mathrm{tot}} .
$$

After the scaling, $H^{\text {sys }}$ and $H^{\text {ex }}$ can be expressed as a power series in $\varepsilon$ :

$$
\begin{aligned}
& H^{\mathrm{sys}}(\boldsymbol{q}, \boldsymbol{p})=\sum_{\nu=0}^{\infty} \varepsilon^{\nu} H_{\nu}^{\mathrm{sys}}(\boldsymbol{q}, \boldsymbol{p}), \\
& H^{\mathrm{ex}}(\boldsymbol{q}, \boldsymbol{p}, t)=\sum_{\nu=0}^{\infty} \varepsilon^{\nu} H_{\nu}^{\mathrm{ex}}(\boldsymbol{q}, \boldsymbol{p}, t),
\end{aligned}
$$

where

$$
\begin{aligned}
& H_{\nu}^{\mathrm{sys}}(\boldsymbol{q}, \boldsymbol{p})=\sum_{\Sigma_{\ell}\left(j_{\ell}+k_{\ell}\right)=\nu+2} \alpha_{j k} q_{1}^{j_{1}} \cdots q_{n}^{j_{n}} p_{1}^{k_{1}} \cdots p_{n}^{k_{n}}, \\
& H_{\nu}^{(\mathrm{ex})}(\boldsymbol{q}, \boldsymbol{p}, t)=\sum_{\Sigma_{\ell}\left(j_{\ell}+k_{\ell}\right)=\nu+1} \beta_{j k}(t) q_{1}^{j_{1}} \cdots q_{n}^{j_{n}} p_{1}^{k_{1}} \cdots p_{n}^{k_{n}} .
\end{aligned}
$$

The total Hamiltonian reads

$$
\begin{aligned}
H^{\mathrm{tot}}(\boldsymbol{q}, \boldsymbol{p}, t) & =H^{\mathrm{sys}}(\boldsymbol{q}, \boldsymbol{p})+H^{\mathrm{ex}}(\boldsymbol{q}, \boldsymbol{p}, t) \\
& =\sum_{\nu=0}^{\infty} \varepsilon^{\nu}\left\{H_{\nu}^{\mathrm{sys}}(\boldsymbol{q}, \boldsymbol{p})+H_{\nu}^{\mathrm{ex}}(\boldsymbol{q}, \boldsymbol{p}, t)\right\} \\
& =H_{0}+\sum_{\nu=1}^{\infty} \varepsilon^{\nu}\left\{H_{\nu}^{\mathrm{sys}}(\boldsymbol{q}, \boldsymbol{p})+H_{\nu}^{\mathrm{ex}}(\boldsymbol{q}, \boldsymbol{p}, t)\right\} .
\end{aligned}
$$

As a consequence of the scaling prescription (6), its leadingorder term

$$
\begin{aligned}
H_{0}(\boldsymbol{q}, \boldsymbol{p}, t)= & H_{0}^{\mathrm{sys}}+H_{0}^{\mathrm{ex}} \\
= & \sum_{\Sigma_{\ell}\left(j_{\ell}+k_{\ell}\right)=2} \alpha_{j k} q_{1}^{j_{1}} \cdots q_{n}^{j_{n}} p_{1}^{k_{1}} \cdots p_{n}^{k_{n}} \\
& +\sum_{\Sigma_{\ell}\left(j_{\ell}+k_{\ell}\right)=1} \beta_{j k}(t) q_{1}^{j_{1}} \cdots q_{n}^{j_{n}} p_{1}^{k_{1}} \cdots p_{n}^{k_{n}}
\end{aligned}
$$

has an autonomous part of degree 2 in coordinates and momenta and a time-dependent part of degree 1 . The associated equations of motion are therefore linear in $\boldsymbol{p}$ and $\boldsymbol{q}$ and have time-dependent driving terms independent of coordinates and momenta. For this situation, although in a nonHamiltonian setting, a time-dependent transition state theory based on an exact solution of the equations of motion was developed in Refs. 19-21. Our treatment of the leading-order Hamiltonian in Secs. II A and II B will be closely patterned after this earlier approach. On the other hand, anharmonicities of the system Hamiltonian and position-dependent couplings to the external fields, both of which cannot be handled within the framework of Refs. 19-21, are relegated to higher-order terms in Eq. (11). A scheme for the perturbative treatment of these terms will be introduced in Sec. II C. It represents a considerable generalization of our earlier method and is the central result of the present paper.

\section{A. Diagonalization of the linearized time-independent dynamics}

As a first step of the normal form procedure, we diagonalize the autonomous part of the leading-order Hamiltonian $H_{0}$ in the standard way. We introduce normal mode coordinates through the linear transformation

$$
\begin{aligned}
Q_{\ell}^{r}= & C_{1}^{(\ell)} q_{1}+C_{2}^{(\ell)} q_{2}+\cdots+C_{n}^{(\ell)} q_{n}+C_{n+1}^{(\ell)} p_{1}+\cdots \\
& +C_{2 N}^{(\ell)} p_{n}, \\
p_{\ell}^{r}= & C_{1}^{(n+\ell)} q_{1}+C_{2}^{(n+\ell)} q_{2}+\cdots+C_{n}^{(n+\ell)} q_{n}+C_{n+1}^{(n+\ell)} p_{1}+\cdots \\
& +C_{2 N}^{(n+\ell)} p_{n},
\end{aligned}
$$

with appropriate coefficients $C_{j}^{(\ell)}$ such that $H_{0}$ takes the form

$$
\begin{aligned}
H_{0}= & \frac{\lambda}{2}\left(P_{1}^{r 2}-Q_{1}^{r 2}\right)+\sum_{\ell=2}^{n} \frac{\omega_{\ell}}{2}\left(P_{\ell}^{r 2}+Q_{\ell}^{r 2}\right)-\sum_{\ell=1}^{n} \xi_{\ell}^{r}(t) Q_{\ell}^{r} \\
& -\sum_{\ell=1}^{n} \eta_{\ell}^{r}(t) P_{\ell}^{r},
\end{aligned}
$$

where $\xi_{\ell}^{r}(t)$ and $\eta_{\ell}^{r}(t)(\ell=1, \ldots, n)$ can be calculated from $\beta_{j k}(t)$ by substituting Eqs. (13) and (14) into Eq. (12). We 
further introduce the coordinates [see Fig. 2(a) for the reactive mode]

$$
\begin{aligned}
& Q_{1}^{c}=\frac{{ }^{\operatorname{def}}}{2_{1}^{r}+P_{1}^{r}}, \quad P_{1}^{c}=\frac{{ }^{\operatorname{def}} P_{1}^{r}-Q_{1}^{r}}{2^{1 / 2}}, \\
& Q_{\ell}^{c}=\frac{Q_{\ell}-i P_{\ell}^{r}}{2^{1 / 2}}, \quad P_{\ell}^{c}=\frac{P_{\ell}{ }^{\operatorname{def}}-i Q_{\ell}^{r}}{2^{1 / 2}} \quad(\ell=2, \ldots, n),
\end{aligned}
$$

which are illustrated in Fig. 2(a) for the reactive mode. For the bath modes, $Q_{\ell}^{c}$ and $P_{\ell}^{c}$ take complex values, whereas $Q_{\ell}^{r}$ and $P_{\ell}^{r}$ are real. This is indicated by the superscripts $r$ and $c$. In these coordinates, $H_{0}$ becomes

$$
H_{0}=\lambda Q_{1}^{c} P_{1}^{c}+\sum_{\ell=2}^{n} i \omega_{\ell} Q_{\ell}^{c} P_{\ell}^{c}-\sum_{\ell=1}^{n} \xi_{\ell}^{c}(t) Q_{\ell}^{c}-\sum_{\ell=1}^{n} \eta_{\ell}^{c}(t) P_{\ell}^{c},
$$

where $\xi_{\ell}^{c}(t), \eta_{\ell}^{c}(t)(\ell=1, \ldots, n)$ are obtained by substituting Eqs. (16) and (17) into Eq. (15).

\section{B. Shift to a time-dependent origin}

To eliminate the time dependence from $H_{0}$, we perform a time-dependent shift of the origin, as suggested in Refs. 19-21 and illustrated in Fig. 2(b):

$$
\begin{aligned}
& \quad \text { def } \\
& q_{\ell}^{c}=Q_{\ell}^{c}-Q_{\ell}^{\ddagger}(t), \\
& \quad \operatorname{def} \\
& p_{\ell}^{c}=P_{\ell}^{c}-P_{\ell}^{\ddagger}(t) \quad(\ell=1, \ldots, n) .
\end{aligned}
$$

The shifts $Q_{\ell}^{\ddagger}$ and $P_{\ell}^{\ddagger}$ are given by

$$
\begin{aligned}
& Q_{1}^{\ddagger}=-S\left[\lambda, \eta_{1}^{c}\right], \\
& P_{1}^{\ddagger}=S\left[-\lambda, \xi_{1}^{c}\right], \\
& Q_{\ell}^{\ddagger}=-S\left[i \omega_{\ell}, \eta_{\ell}^{c}\right], \\
& P_{\ell}^{\ddagger}=S\left[-i \omega_{\ell}, \xi_{\ell}^{c}\right] \quad(\ell=2, \ldots, n),
\end{aligned}
$$

where the symbol $S[\cdot, \cdot]$ is defined as follows. For a function $f(t)$ with the Fourier transform

$$
\begin{gathered}
\hat{f}(\omega)=\frac{1}{(2 \pi)^{1 / 2}} \int_{-\infty}^{+\infty} f(t) \exp (-i \omega t) d t, \\
f(t)=\frac{1}{(2 \pi)^{1 / 2}} \int_{-\infty}^{+\infty} \hat{f}(\omega) \exp (i \omega t) d \omega,
\end{gathered}
$$

and for a complex number $\mu$,

$$
S[\mu, f](t)=\frac{1}{(2 \pi)^{1 / 2}} \int_{-\infty}^{+\infty} \frac{\hat{f}(\omega)}{-\mu+i \omega} \exp (i \omega t) d \omega .
$$

If $\operatorname{Re} \mu \neq 0$, the $S$-functional can be written explicitly as in Ref. 20:

$$
S[\mu, f](t)= \begin{cases}-\int_{t}^{\infty} f(\tau) \exp (\mu(t-\tau)) d \tau & : \operatorname{Re} \mu>0 \\ +\int_{-\infty}^{t} f(\tau) \exp (\mu(t-\tau)) d \tau & : \operatorname{Re} \mu<0 .\end{cases}
$$

Therefore, the components $Q_{1}^{\ddagger}(t)$ and $P_{1}^{\ddagger}(t)$ correspond to the TS trajectory of Refs. 19-21, which was defined as a particular solution of the lowest-order equations of motion that remains in the vicinity of the barrier for all times. If $f(t)$ describes a short pulse, i.e., $f(t) \rightarrow 0$ as $t \rightarrow \pm \infty$, it is clear from Eq. (28) that $S[\mu, f](t) \rightarrow 0$ as $t \rightarrow \pm \infty$, as is required for the components of the TS trajectory.

If, however, $\mu=i \omega_{0}$ is purely imaginary, the integral in Eq. (27) is ill defined because the integrand has a pole on the integration path. This divergence must be regularized to yield a suitable expression for the TS trajectory. An obvious way to do this is to add an infinitesimal real part to the eigenvalues, i.e., to replace the $S$ functional in Eqs. (23) and (24) by

$$
S_{ \pm}\left[i \omega_{0}, f\right](t)=S\left[i \omega_{0} \pm \epsilon, f\right], \quad \epsilon>0 .
$$

These regularizations are well defined if the Fourier transform $\hat{f}(\omega)$ is regular at $\omega=\omega_{0}$. They differ in the boundary conditions they satisfy: By Eq. (28), $S_{+}\left[i \omega_{0}, f\right](t)$ tends to zero as $t \rightarrow+\infty$. As $t \rightarrow-\infty$,

$$
\begin{aligned}
S_{+}\left[i \omega_{0}, f\right](t) & \rightarrow-\exp \left(i \omega_{0} t\right) \int_{-\infty}^{\infty} f(\tau) \exp \left(-i \omega_{0} \tau\right) \\
& =-(2 \pi)^{1 / 2} \hat{f}\left(\omega_{0}\right) \exp \left(i \omega_{0} t\right) .
\end{aligned}
$$

Conversely, $S_{-}\left[i \omega_{0}, f\right](t) \rightarrow 0$ as $t \rightarrow-\infty$, and $S_{-}\left[i \omega_{0}, f\right](t)$ $\rightarrow(2 \pi)^{1 / 2} \hat{f}\left(\omega_{0}\right) \exp \left(i \omega_{0} t\right)$ as $t \rightarrow+\infty$. Both regularizations remain bounded for all times and are therefore equally suitable as components of the TS trajectory. We thus find that in the presence of purely imaginary eigenvalues the TS trajectory is no longer uniquely defined. Dynamically speaking, imaginary eigenvalues correspond to undamped oscillations. The motion in these modes always remains bounded for all times, so that the requirement that the TS trajectory must never leave the vicinity of the barrier does not single out a specific trajectory. In the bath modes, we are therefore free to pick an arbitrary trajectory and designate it as the TS trajectory. By convention, we will choose the symmetrized version,

$$
S\left[i \omega_{0}, f\right](t)=\frac{\operatorname{def}}{2}\left(S_{+}\left[i \omega_{0}, f\right](t)+S_{-}\left[i \omega_{0}, f\right](t)\right),
$$

which corresponds to replacing the integral in Eq. (27) by its principal value. With this definition, Eqs. (21)-(24) specify a TS trajectory if the Fourier spectra of the driving forces $\xi_{\ell}^{c}(t)$ and $\eta_{\ell}^{c}(t)$ are smooth, which is true for realistic laser pulses.

For functions $f(t), g(t)$ and constants $a, b, \mu, \nu$, the following properties of the $S$ symbol can readily be shown:

$$
S[\mu, a f+b g]=a S[\mu, f]+b S[\mu, g],
$$

$$
\left(\frac{d}{d t}-\mu\right) S[\mu, f]=f,
$$




$$
\begin{aligned}
& S[\mu, S[\lambda, f]]=S[\lambda, S[\mu, f]]=\frac{1}{\mu-\lambda}\{S[\mu, f]-S[\lambda, f]\} \\
& S[\mu, 1]=-\frac{1}{\mu}, \\
& \text { If } \int_{-\infty}^{+\infty} f(t) d t=0 \text { then } \int_{-\infty}^{+\infty} S[\mu, f](t) d t=0 .
\end{aligned}
$$

If $f(t)$ is proportional to an electric field strength, as for the dipole coupling (5), the hypothesis of Eq. (36), i.e., $\hat{f}(0)=0$, is always satisfied as a consequence of Maxwell's equations. $^{16}$

The shift (19) and (20) to the TS trajectory as a timedependent origin is described by the generating function

$$
F\left(\boldsymbol{q}^{c}, \boldsymbol{P}^{c}, t\right)=\sum_{\ell=1}^{n}\left\{q_{\ell}^{c} P_{\ell}^{c}-q_{\ell}^{c} P_{\ell}^{\ddagger}(t)+Q_{\ell}^{\ddagger}(t) P_{\ell}^{c}\right\} .
$$

The new Hamiltonian $\tilde{H}\left(\boldsymbol{q}^{c}, \boldsymbol{p}^{c}, t\right)$ is given by

$$
\begin{aligned}
\tilde{H}\left(\boldsymbol{q}^{c}, \boldsymbol{p}^{c}, t\right) & =H^{\mathrm{tot}}-\frac{\partial F}{\partial t} \\
& =H_{0}-\frac{\partial F}{\partial t}+\sum_{\nu=1}^{\infty} \varepsilon^{\nu}\left\{H_{\nu}^{\mathrm{sys}}+H_{\nu}^{\mathrm{ex}}\right\} .
\end{aligned}
$$

A simple calculation using Eq. (33) shows that

$$
\begin{aligned}
H_{0}-\frac{\partial F}{\partial t}= & \lambda q_{1}^{c} p_{1}^{c}-\xi_{1}(t) Q_{1}^{\ddagger}(t)+\sum_{\ell=2}^{n}\left\{i \omega_{\ell} q_{\ell}^{c} p_{\ell}^{c}\right. \\
& \left.+\xi_{\ell}(t) Q_{\ell}^{\ddagger}(t)\right\} .
\end{aligned}
$$

Terms independent of $p_{\ell}^{c}$ and $q_{\ell}^{c}$ have no influence on the equations of motion and can be omitted. We then obtain a Hamiltonian [that will not be confused with the original Hamiltonian (2)]

$$
H\left(\boldsymbol{q}^{c}, \boldsymbol{p}^{c}, t\right)=H_{0}^{(0)}\left(\boldsymbol{q}^{c}, \boldsymbol{p}^{c}\right)+\sum_{\nu=1}^{\infty} \varepsilon^{\nu} H_{\nu}\left(\boldsymbol{q}^{c}, \boldsymbol{p}^{c}, t\right)
$$

where the harmonic part

$$
H_{0}^{(0)}\left(\boldsymbol{q}^{c}, \boldsymbol{p}^{c}\right)=\lambda q_{1}^{c} p_{1}^{c}+\sum_{\ell=2}^{n} i \omega_{\ell} q_{\ell}^{c} p_{\ell}^{c}
$$

is formally the same as the autonomous part of the leadingorder Hamiltonian $H_{0}$ in Eq. (19) and the higher-order terms $H_{\nu}\left(\boldsymbol{q}^{c}, \boldsymbol{p}^{c}, t\right)=H_{\nu}^{\mathrm{sys}}+H_{\nu}^{\mathrm{ex}}$ are the same as in Eq. (11) but rewritten in the new coordinates.

The dynamics of the harmonic part $H_{0}^{(0)}$ can be solved exactly. It conserves the action variables $I_{1}=q_{1}^{c} p_{1}^{c}$, and $I_{\ell}$ $=i q_{\ell}^{c} p_{\ell}^{c}(\ell=2, \ldots, n)$. In the next section, we will incorporate the effect of the anharmonic terms $H_{\nu}(\nu=1,2, \ldots)$ by regarding them as perturbations to the integrable system $H_{0}^{(0)}$.

\section{Time-dependent normal form theory}

This section describes a final nonlinear canonical transformation $\left(\boldsymbol{q}^{c}, \boldsymbol{p}^{c}\right) \mapsto\left(\overline{\boldsymbol{q}}^{c}, \overline{\boldsymbol{p}}^{c}\right)$ illustrated in Fig. 2(c). This transformation will be chosen such as to decouple the reaction coordinate from the bath modes. To this end, we will use time-dependent Lie transformations ${ }^{22}$ in the formulation of Dragt and Finn. ${ }^{27}$ First we extend the phase space from $\left(\boldsymbol{q}^{c}, \boldsymbol{p}^{c}\right)$ to $\left(\boldsymbol{q}^{c}, \tau, \boldsymbol{p}^{c}, P_{\tau}\right)$ with Hamiltonian

$$
K\left(\boldsymbol{q}^{c}, \tau, \boldsymbol{p}^{c}, P_{\tau}\right)=H\left(\boldsymbol{q}^{c}, \boldsymbol{p}^{c}, \tau\right)+P_{\tau},
$$

where the canonical coordinate $\tau$ takes the same value as $t$ and $P_{\tau}$ is its conjugate momentum. The Taylor expansion of $K$ is given by

$$
K\left(\boldsymbol{q}^{c}, \tau, \boldsymbol{p}^{c}, P_{\tau}\right)=K_{0}\left(\boldsymbol{q}^{c}, \tau, \boldsymbol{p}^{c}, P_{\tau}\right)+\sum_{\nu=1}^{\infty} \varepsilon^{\nu} K_{\nu}\left(\boldsymbol{q}^{c}, \tau, \boldsymbol{p}^{c}\right),
$$

where

$$
\begin{aligned}
K_{0}\left(\boldsymbol{q}^{c}, \tau, \boldsymbol{p}^{c}, P_{\tau}\right) & =H_{0}^{(0)}\left(\boldsymbol{q}^{c}, \boldsymbol{p}^{c}, \tau\right)+P_{\tau} \\
& =\lambda q_{1}^{c} p_{1}^{c}+\sum_{\ell=2}^{n} i \omega_{\ell} q_{\ell}^{c} p_{\ell}^{c}+P_{\tau},
\end{aligned}
$$

$$
K_{\nu}\left(\boldsymbol{q}^{c}, \tau, \boldsymbol{p}^{c}\right)=H_{\nu}\left(\boldsymbol{q}^{c}, \boldsymbol{p}^{c}, \tau\right) \quad \text { for } \nu \geqslant 1 .
$$

We will now construct a canonical transformation $\left(\boldsymbol{q}^{c}, \tau, \boldsymbol{p}^{c}, P_{\tau}\right) \mapsto\left(\overline{\boldsymbol{q}}^{c}, \tau, \overline{\boldsymbol{p}}^{c}, P_{\tau}\right)$ that leaves the time coordinate $\tau$ unchanged and that decouples the reactive mode from the bath modes to an arbitrarily high order $N$ of perturbation theory. It will be given by a sequence of Lie transformations:

$$
\begin{aligned}
& \bar{q}_{\ell}^{c}=\exp \left(-\varepsilon F_{1}\right) \exp \left(-\varepsilon^{2} F_{2}\right) \cdots \exp \left(-\varepsilon^{N} F_{N}\right) q_{\ell}^{c}, \\
& \bar{p}_{\ell}^{c}=\exp \left(-\varepsilon F_{1}\right) \exp \left(-\varepsilon^{2} F_{2}\right) \cdots \exp \left(-\varepsilon^{N} F_{N}\right) p_{\ell}^{c},
\end{aligned}
$$

where

$$
F_{\nu}=\left\{\cdot, f_{\nu}\right\}
$$

is the operation of Poisson bracket with a function $f_{\nu}$ that will be specified below. The Hamiltonian $K$ is transformed into

$$
\bar{K}=\exp \left(\varepsilon^{N} F_{N}\right) \cdots \exp \left(\varepsilon^{2} F_{2}\right) \exp \left(\varepsilon F_{1}\right) K
$$

It is now our goal to find functions $f_{\nu}$ that achieve the desired decoupling in $\bar{K}$.

If we define a sequence of partially transformed Hamiltonians $\bar{K}^{(\mu)}=\sum_{\nu=0}^{\infty} \varepsilon^{\nu} \bar{K}_{\nu}^{(\mu)}$ by $\bar{K}^{(0)}=K$ and

$$
\begin{aligned}
\bar{K}^{(\mu)} & =\exp \left(\varepsilon^{\mu} F_{\mu}\right) \bar{K}^{(\mu-1)} \\
& =\exp \left(\varepsilon^{\mu} F_{\mu}\right) \cdots \exp \left(\varepsilon^{2} F_{2}\right) \exp \left(\varepsilon F_{1}\right) K,
\end{aligned}
$$

we find the recursion formulas

$$
\begin{aligned}
& \nu<\mu: \bar{K}_{\nu}^{(\mu)}=\bar{K}_{\nu}^{(\mu-1)}, \\
& \nu=\mu: \bar{K}_{\mu}^{(\mu)}=\bar{K}_{\mu}^{(\mu-1)}+F_{\mu} \bar{K}_{0}^{(0)},
\end{aligned}
$$




$$
\nu>\mu: \bar{K}_{\nu}^{(\mu)}=\bar{K}_{\nu}^{(\mu-1)}+\sum_{s=1}^{\infty} \frac{\left(F_{\mu}\right)^{s}}{s !} \bar{K}_{\nu-s \mu}^{(\mu-1)}
$$

Because the term of the order $\nu$ is unchanged for $\mu>\nu$, in the final Hamiltonian $\bar{K}=\bar{K}^{(N)}$ it reads

$$
\bar{K}_{\nu}=\bar{K}_{\nu}^{(N)}=\bar{K}_{\nu}^{(N-1)}=\cdots=\bar{K}_{\nu}^{(\nu)}=\bar{K}_{\nu}^{(\nu-1)}+F_{\nu} \bar{K}_{0}^{(0)} .
$$

Thus, $f_{\nu}$ should be chosen so that the decoupling of the reactive mode is achieved in the term $K_{\nu}^{(\nu)}$.

In the present case,

$$
\begin{aligned}
\bar{K}_{\nu}^{(\nu-1)}\left(\boldsymbol{q}^{c}, \boldsymbol{p}^{c}\right)= & \sum_{j, k} h_{j k}^{(\nu)}(\tau)\left(\bar{q}_{1}^{c}\right)^{j_{1}}\left(\bar{q}_{2}^{c}\right)^{j_{2}} \cdots\left(\bar{q}_{n}^{c}\right)^{j_{n}} \\
& \times\left(\bar{p}_{1}^{c}\right)^{k_{1}}\left(\bar{p}_{2}^{c}\right)^{k_{2}} \cdots\left(\bar{p}_{n}^{c}\right)^{k_{n}}
\end{aligned}
$$

is a polynomial, where $h_{j k}^{(\nu)}(\tau)$ are coefficients that depend on $\tau$. We can also express $f_{\nu}$ as a polynomial

$$
f_{\nu}=\sum_{j, k} w_{\nu, j k}(\tau)\left(\bar{q}_{1}^{c}\right)^{j_{1}}\left(\bar{q}_{2}^{c}\right)^{j_{2}} \cdots\left(\bar{q}_{n}^{c}\right)^{j_{n}}\left(\bar{p}_{1}^{c}\right)^{k_{1}}\left(\bar{p}_{2}^{c}\right)^{k_{2}} \cdots\left(\bar{p}_{n}^{c}\right)^{k_{n}},
$$

containing the same monomials $j k$ that occur in Eq. (56) With $\bar{K}_{0}^{(0)}$ given by Eq. (45), Eq. (55) yields

$$
\begin{aligned}
\bar{K}_{\nu}^{(N)}= & \sum_{j, k}\left[h_{j k}^{(\nu)}(\tau)-\left(\frac{d}{d \tau}-\gamma_{j k}\right) w_{\nu, j k}\right] \\
& \times\left(\bar{q}_{1}^{c}\right)^{j_{1}}\left(\bar{q}_{2}^{c}\right)^{j_{2}} \cdots\left(\bar{q}_{n}^{c}\right)^{j_{n}}\left(\bar{p}_{1}^{c}\right)^{k_{1}}\left(\bar{p}_{2}^{c}\right)^{k_{2} \cdots\left(\bar{p}_{n}^{c}\right)^{k_{n}},}
\end{aligned}
$$

where

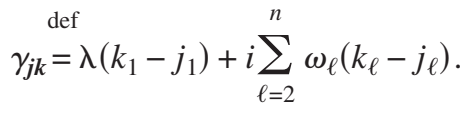

Thus, by setting

$$
w_{\nu, j k}=S\left[\gamma_{j k}, h_{j k}^{(\nu)}\right],
$$

we can eliminate the term $j k$ from $\bar{K}^{(\nu)}$ [see Eq. (33)]. If $h_{j k}^{(\nu)}$ does not depend on $\tau$, we obtain

$$
w_{\nu, j k}=\frac{h_{j k}^{(\nu)}}{\gamma_{j k}},
$$

which is well known in the theory of time-independent $\mathrm{NF}^{1-3}$

In the calculation of the coefficients in Eq. (60), once again one has to pay attention to convergence. If $\gamma_{j k}$ is purely imaginary, the integrand in the definition (27) has a singularity on the real axis. It was noted in Sec. II B that taking the principal value circumvents this problem for the calculation of the TS trajectory because the Fourier transform of the laser pulse is regular. However, the Fourier transform of the coefficients $h_{j k}^{(\nu)}$ may be singular there because through Eqs. (19) and (20) these coefficients depend on the TS trajectory, the Fourier transform of which has a pole. Thus, the $S$ functional in Eq. (60) may diverge for purely imaginary $\gamma_{j k}$. This effect can be interpreted as due to a resonance between a bath mode and the laser pulse. It prevents us from eliminat-

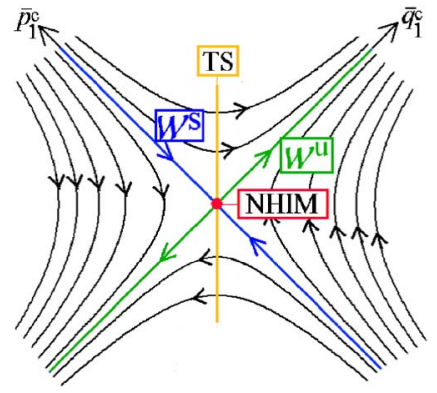

FIG. 3. (Color online) Phase space structures projected onto the NF reactive mode (schematic). The action $\bar{I}_{1}=\bar{q}_{1}^{c} \bar{p}_{1}^{c}$ is conserved and restricts trajectories to the hyperbolas shown in black.

ing the resonant term from the Hamiltonian and thereby from constructing a coordinate system in which all degrees of freedom decouple.

A resonance that makes $\gamma_{j k}$ purely imaginary can only occur if $j_{1}=k_{1}$. We propose to retain all such terms in the normal form Hamiltonian and to eliminate all terms with $j_{1}$ $\neq k_{1}$. Such a partial normalization avoids resonances, which makes it a powerful tool in many applications (see, e.g., Refs. 1, 8, 28, and 29).

The transformation functions $f_{\nu}$ in (57) are independent of $P_{\tau}$, so that the time coordinate

$$
\bar{\tau}=\exp \left(-\varepsilon F_{1}\right) \exp \left(-\varepsilon^{2} F_{2}\right) \cdots \exp \left(-\varepsilon^{N} F_{N}\right) \tau=\tau
$$

is unchanged under the transformation, as desired. In addition, because of the special form of $K_{\nu}^{(\mu)}$ in Eqs. (45) and (46), $F_{\mu} K_{\nu}^{(0)}=\left\{K_{\nu}^{(0)}, f_{\mu}\right\}$ is independent of $P_{\tau}$ for all $\mu$ and $\nu$. Therefore, the normal form Hamiltonian $\bar{K}$ depends on $P_{\tau}$ only through its lowest-order term $\bar{K}_{0}=K_{0}$. We can thus return to a time-dependent formulation in the nonextended phase space with the normal form Hamiltonian

$$
\begin{aligned}
\bar{H}\left(\overline{\boldsymbol{q}}^{c}, \overline{\boldsymbol{p}}^{c}, t\right) & =\bar{K}\left(\overline{\boldsymbol{q}}^{c}, t, \overline{\boldsymbol{p}}^{c}, \bar{P}_{\tau}\right)-\bar{P}_{\tau} \\
& =H_{0}^{(0)}\left(\overline{\boldsymbol{q}}^{c}, \overline{\boldsymbol{p}}^{c}\right)+\sum_{\nu=1}^{\infty} \varepsilon^{\nu} \bar{K}_{\nu}\left(\overline{\boldsymbol{q}}^{c}, t, \overline{\boldsymbol{p}}^{c}\right),
\end{aligned}
$$

where we write $t$ again instead of the time coordinate $\tau$.

Because the normal form Hamiltonian contains only terms with $j_{1}=k_{1}$, it takes the form

$$
\begin{aligned}
\bar{H}\left(\overline{\boldsymbol{q}}^{c}, \overline{\boldsymbol{p}}^{c}, t\right)= & \lambda \bar{q}_{1}^{c} \bar{p}_{1}^{c}+\sum_{\ell=2}^{n} i \omega_{\ell} \bar{q}_{\ell}^{c} \bar{p}_{\ell}^{c}+\sum_{j, k} \bar{a}_{j k}^{(\nu)}(t) \\
& \times\left(\bar{q}_{1}^{c} \bar{p}_{1}^{c}\right)^{j_{1}}\left(\bar{q}_{2}^{c}\right)^{j_{2}} \cdots\left(\bar{q}_{n}^{c}\right)^{j_{n}}\left(\bar{p}_{2}^{c}\right)^{k_{2}} \cdots\left(\bar{p}_{n}^{c}\right)^{k_{n}},(64)
\end{aligned}
$$

where it is understood that terms of order larger than $N$, which are not in normal form, should be dropped. For the Hamiltonian (64), the action of the reactive mode $\bar{I}_{1}=\bar{q}_{1}^{c} \bar{p}_{1}^{c}$ is conserved. This reflects the desired separation of the reactive mode from the bath modes. When projected onto the $\left(\bar{q}_{1}^{c}, \bar{p}_{1}^{c}\right)$ plane, trajectories follow a hyperbola, as depicted in Fig. 3. Trajectories with $\bar{I}_{1}>0$ are (forward or backward) reactive, those with $\bar{I}_{1}<0$ are not. The separatrices between reactive and nonreactive trajectories are thus given by $\bar{I}_{1}=0$. 
Through the normal form coordinates, geometrical objects such as the NHIM $\mathcal{M}$, its stable manifold $\mathcal{W}^{s}$ and unstable manifold $\mathcal{W}^{u}$, and the transition state dividing surface $\mathcal{T}$ can be defined in a way analogous to that of Refs. 1 and 2 (see Fig. 3):

$$
\begin{aligned}
& \text { def } \\
& \mathcal{M}=\left\{\left(\overline{\boldsymbol{q}}^{c}, \overline{\boldsymbol{p}}^{c}, t\right) \mid\left(\overline{\boldsymbol{q}}^{c}, \overline{\boldsymbol{p}}^{c}, t\right) \in \Omega, \quad \bar{q}_{1}^{c}=\bar{p}_{1}^{c}=0\right\}, \\
& \text { def } \\
& \mathcal{W}^{s}=\left\{\left(\overline{\boldsymbol{q}}^{c}, \overline{\boldsymbol{p}}^{c}, t\right) \mid\left(\overline{\boldsymbol{q}}^{c}, \overline{\boldsymbol{p}}^{c}, t\right) \in \Omega, \quad \bar{q}_{1}^{c}=0\right\}, \\
& \text { def } \\
& \mathcal{W}^{u}=\left\{\left(\overline{\boldsymbol{q}}^{c}, \overline{\boldsymbol{p}}^{c}, t\right) \mid\left(\overline{\boldsymbol{q}}^{c}, \overline{\boldsymbol{p}}^{c}, t\right) \in \Omega, \quad \bar{p}_{1}^{c}=0\right\}, \\
& \mathcal{T}^{\operatorname{def}}\left\{\left(\overline{\boldsymbol{q}}^{c}, \overline{\boldsymbol{p}}^{c}, t\right) \mid\left(\overline{\boldsymbol{q}}^{c}, \overline{\boldsymbol{p}}^{c}, t\right) \in \Omega, \quad \bar{q}_{1}^{r}=\frac{\bar{q}_{1}^{c}-\bar{p}_{1}^{c}}{2^{1 / 2}}=0\right\} .
\end{aligned}
$$

These definitions are valid locally within the region of convergence $\Omega$ of the NF expansion. They can be put in the original coordinates by inverting the nonlinear transformation [Eqs. (47) and (48)], the shift [Eqs. (19) and (20)], and then the linear transformations [Eqs. (13), (14), (16), and (17)]. The stable and unstable manifolds can be continued numerically beyond the region $\Omega$ as in the autonomous setting. ${ }^{4,5}$ The dimensions of $\mathcal{M}, \mathcal{W}^{s}, \mathcal{W}^{u}$, and $\mathcal{T}$ are $2 n-1$, $2 n, 2 n$, and $2 n$, respectively, in the $(2 n+1)$-dimensional phase space (including time). These dimensions are increased by 2 compared to the time-independent case because the manifolds are time dependent and are not confined to an energy shell.

The normal form procedure requires the truncation of the Hamiltonian to a finite expansion order $N$. It is therefore important to monitor the error caused by the truncation and the convergence of the expansion with increasing $N$. In Ref. 30 , we suggested to use the energy error for this purpose in an autonomous system. Here we will use an alternative criterion that is more directly related to the equations of motion, that is, the error of Hamiltonian vector field. We monitor the Euclidean norm of the difference of the vector fields calculated by the original Hamiltonian and the TDNF Hamiltonian:

$$
\begin{aligned}
\Delta v= & {\left[\sum_{\ell=1}^{\operatorname{def}}\left[\frac{d}{d t} \bar{q}_{\ell}^{c}\left(\boldsymbol{q}^{c}(t), \boldsymbol{p}^{c}(t), t\right)-\frac{\partial \bar{H}}{\partial \bar{p}_{\ell}^{c}}\right)^{2}\right.} \\
& \left.+\sum_{\ell=1}^{n}\left(\frac{d}{d t} \bar{p}_{\ell}^{c}\left(\boldsymbol{q}^{c}(t), \boldsymbol{p}^{c}(t), t\right)+\frac{\partial \bar{H}}{\partial \bar{q}_{\ell}^{c}}\right)^{2}\right]^{1 / 2} .
\end{aligned}
$$

Here, $\bar{q}_{\ell}^{c}\left(\boldsymbol{q}^{c}(t), \boldsymbol{p}^{c}(t), t\right)$ and $\bar{p}_{\ell}^{c}\left(\boldsymbol{q}^{c}(t), \boldsymbol{p}^{c}(t), t\right)$ mean $\bar{q}_{\ell}^{c}$ and $\bar{p}_{\ell}^{c}$ as function of the original coordinates $\left(\boldsymbol{q}^{c}, \boldsymbol{p}^{c}\right)$, with $\boldsymbol{q}^{c}(t)$ and $\boldsymbol{p}^{c}(t)$ calculated by trajectory simulation with the original Hamiltonian. The Hamiltonian vector field $\left(\partial \bar{H} / \partial \bar{p}_{\ell}^{c}, \partial \bar{H} / \partial \bar{q}_{\ell}^{c}\right)$ is calculated by the transformed Hamiltonian $\bar{H}$. If $\left(\boldsymbol{q}^{c}, \boldsymbol{p}^{c}\right)$ is in the convergence range, and if the NF expansion is taken up to the infinite order, then $\Delta v$ becomes zero. Thus, we use a decrease in the value of $\Delta v$ as a numerical criterion for convergence. To judge whether the error $\Delta v$ is small, we compare it with the norm $v$ of the Hamiltonian vector field:

$$
v=\left[\sum_{\ell=1}^{n}\left(\frac{\partial \bar{H}}{\partial \bar{p}_{\ell}^{c}}\right)^{2}+\sum_{\ell=1}^{n}\left(\frac{\partial \bar{H}}{\partial \bar{q}_{\ell}^{c}}\right)^{2}\right]^{1 / 2} .
$$

\section{THE DRIVEN HÉNON-HEILES SYSTEM}

In this section we will apply the theory developed in the preceding section to an externally driven Hénon-Heiles system, which serves as a simple model for a reaction with several open channels. Through numerical trajectory calculations we find that the set of trajectories that lead into any given channel possesses a structure reminiscent of the "reactive islands" known in autonomous systems. ${ }^{4,6,11-13}$ The time-dependent invariant manifolds introduced here form the boundaries of the reactive islands and thus provide a geometric interpretation of the island structure. Time-dependent normal form theory will prove to be an effective tool for their calculation.

The Hénon-Heiles potential ${ }^{26}$ has one minimum at the origin and three saddle points $S_{R}=\left(3^{1 / 2} / 2,-1 / 2\right)$, $S_{A}=\left(-3^{1 / 2} / 2,-1 / 2\right)$, and $S_{B}=(0,1)$. They separate a central region from three asymptotic regions (see Fig. 1) that we interpret as defining a reactant channel and two product channels A and B. The central region corresponds to an intermediate activated complex. As pointed out in Sec. I, this model captures salient features of multichannel chemical reactions. In the absence of external driving, the three saddles are equivalent. Their barrier height relative to the origin is $1 / 6$. Their normal mode frequencies are, in the notation of Eq. (18), $\lambda=1$ for the reactive mode and $\omega_{2}=3^{1 / 2}$ for the bath mode.

In Ref. 31, it was found that the interaction between reacting molecules and a laser field is much larger for the activated complex than for the isolated molecules. We assume this result to be typical of many molecular systems. This finding motivates us to model the coupling of the molecular system to the laser field by a Gaussian that is peaked at the origin. We are thus led to the Hamiltonian

$$
\begin{aligned}
H= & \frac{1}{2}\left(p_{x}^{2}+p_{y}^{2}\right)+\frac{1}{2}\left(x^{2}+y^{2}\right)+x^{2} y-\frac{1}{3} y^{3} \\
& +\mathcal{E}_{1}(t) \exp \left(-\alpha x^{2}-\beta y^{2}\right) .
\end{aligned}
$$

Here, $x$ and $y$ are position coordinates and $p_{x}$ and $p_{y}$ are conjugate momenta. $\mathcal{E}_{1}(t)$ denotes the electric field, and $\alpha$ and $\beta$ are parameters that introduce an asymmetry among the asymptotic regions. Since this is a model calculation, we use a unit system in which the vibrational frequency at the origin is scaled to 1 . We use $\alpha=2$ and $\beta=4$ in what follows. The laser-molecule interaction is proportional to $\mathcal{E}_{1}(t)$, thus representing a dipole interaction. Inclusion of the polarizability would result in terms proportional to the square of $\mathcal{E}_{1}(t) .{ }^{31}$ by $^{32}$

We use the driving field $\mathcal{E}_{1}(t)$ shown in Fig. 4 and given

$$
\mathcal{E}_{1}(t)=-\frac{\partial}{\partial t} A(t),
$$




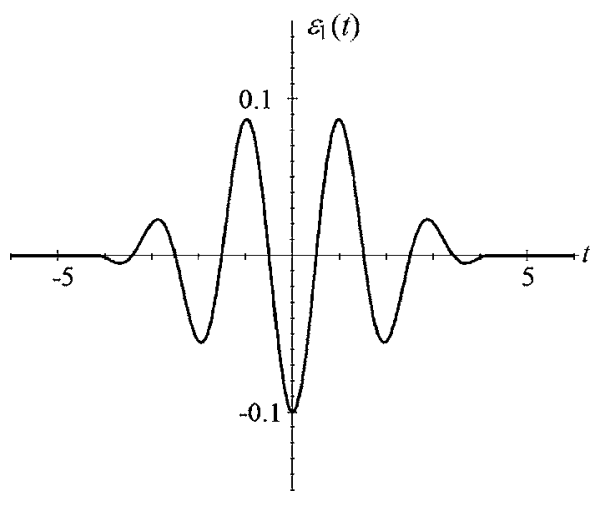

FIG. 4. The electric field (72) used to drive the Hénon-Heiles system.

$$
A(t)=\left\{\begin{array}{cc}
-A_{0} \cos ^{2}\left(\frac{\omega t}{2 N}\right) \sin (\omega t+\phi) & \left(\text { for }|t|<\frac{N \pi}{\omega}\right) \\
0 & \text { (otherwise) } .
\end{array}\right.
$$

The parameters $\omega$ and $A_{0}$ are the laser frequency and the amplitude, respectively. The phase $\phi$ is called the carrierenvelope phase. ${ }^{14,32}$ The parameter $N$ is the number of cycles contained under the envelope. This pulse satisfies the zeroarea condition $\int_{-\infty}^{+\infty} \mathcal{E}_{1}(t) d t=0$. We use $\omega=3, A_{0} \omega=0.1, N=4$, and $\phi=\pi$, which we found to exhibit the effect of the laser field most clearly. Thus, the laser frequency $\omega$ is three times the "molecular" frequency $\omega_{0}=1$ of small vibrations around the origin. With this choice of parameters, the external field is zero for $|t|>4.189$.

We sample initial conditions for our numerical trajectory study on the surface defined by

$$
3^{1 / 2} x-y=3,
$$

at the initial time $t_{0}=-4.2$, just before the onset of the pulse, and with the initial energy $E_{0}=0.3$. (Note that the energy is well defined before the pulse starts.) These initial conditions can be specified by the parameters

$$
\begin{aligned}
& { }^{\text {def }}=\frac{x+3^{1 / 2} y}{2}, \\
& \quad{ }^{\text {def }} p_{x}+3^{1 / 2} p_{y} \\
& 2
\end{aligned}
$$

which coincide with the canonical normal mode coordinates of the bath mode at $S_{R}$.

An example of the unit conversion from our scaled units to conventional units can be given as follows: One of length, mass, and time in our units may correspond to 1.6 a.u. $(=0.84 \AA), \quad 1837$ a.u. $\quad\left(=1.7 \times 10^{-27} \mathrm{~kg}\right), \quad$ and $\quad 220$ a.u. $(=5.3 \mathrm{fs})$, near the time scale of proton motion $(\sim 7 \mathrm{fs})$, respectively. This makes the unit of energy 0.096 a.u. $(=60 \mathrm{kcal} / \mathrm{mol})$. Thus the barrier height $(1 / 6$ in our scaled units) becomes 0.016 a.u. $=10 \mathrm{kcal} / \mathrm{mol}$, which is in the right order of the realistic systems. ${ }^{33,34}$ The harmonic frequency at the center becomes $220^{-1}=0.0046$ a.u., corresponding to
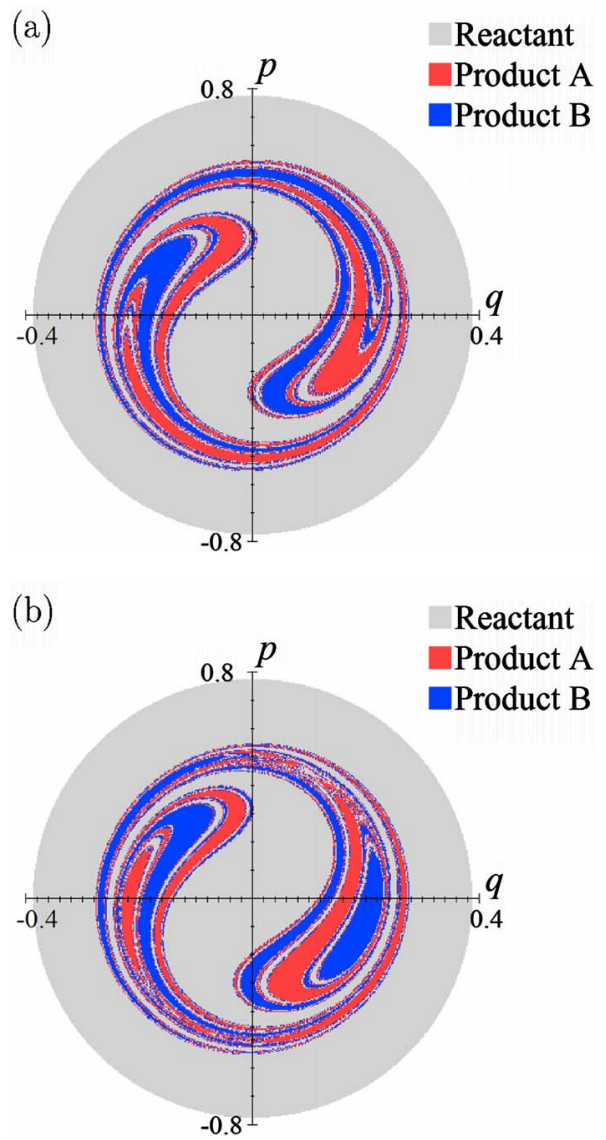

FIG. 5. (Color online) Initial conditions that result in product A, product B, or a return into the reactant channel are shown by medium (red), dark (blue), and light (gray) colors. (a) Without an external field. (b) With the driving field (72). The external field distorts the pattern of islands and breaks the symmetry between channels A and B.

$1000 \mathrm{~cm}^{-1}$. Thus, our initial energy of 0.3 , which corresponds to 0.029 a.u. $\left(=18 \mathrm{kcal} / \mathrm{mol}=6300 \mathrm{~cm}^{-1}\right)$, is larger than the zero point energy of $500 \mathrm{~cm}^{-1}$.

As described above, the outcome of a reaction is determined by the channel in which a trajectory finally leaves the interaction region. Figure 5 shows the final channel as a function of the initial condition $(q, p)$. In the absence of the external field, as shown in Fig. 5(a), there is a symmetry between the channels A and B: The reflection $(q, p) \mapsto(-q$, $-p$ ) interchanges their roles, as should be expected from the symmetry of the Hénon-Heiles system. The time-dependent driving, which is anisotropic because $\alpha \neq \beta$ in Eq. (71), breaks this symmetry, as can clearly be seen in Fig. 5(b). There is an asymmetric shift of the phase space regions that lead into different final channels, as can most clearly be seen in the regions around $(q, p)=( \pm 0.22,0)$. In addition, the branching ratio, that is, the total production of $\mathrm{B}$ divided by that of $\mathrm{A}$, is increased to 1.075 . This is precisely the effect that needs to be understood in detail if one wishes to control the outcome of a reaction through a suitably tailored laser pulse.

The most conspicuous feature of Fig. 5 is the existence of several different regions, or "islands," of initial conditions that lead into the same final channel and that intertwine with the islands of the other channels in a complicated manner. As 
(a)

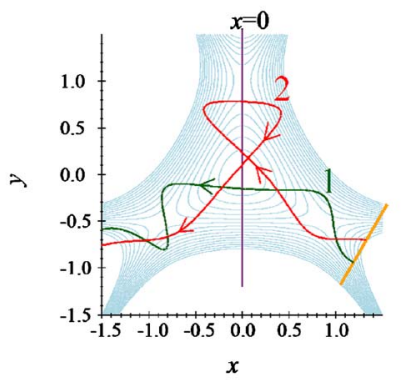

(c)

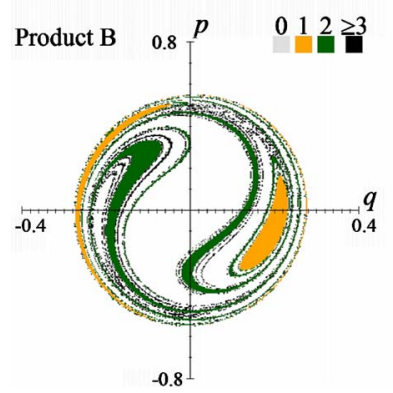

(b)

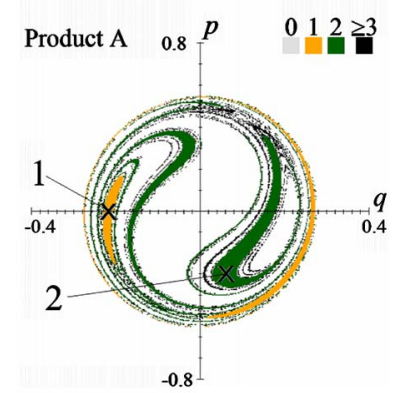

(d)

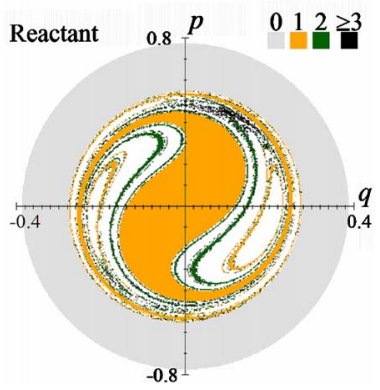

FIG. 6. (Color online) (a) Trajectories that reach the same final channel from different islands show qualitatively different behaviors. They can be distinguished by the number of crossings with the dividing line $L_{A}: x=0$. (b) Number of crossings with $L_{A}: x=0$ for trajectories with final state $A$. Only crossings from positive to negative $x$ are counted. Trajectories within one island share the same number of crossings. The two initial conditions used to draw the trajectories in panel (a) are marked by crossed symbols. (c) Number of crossings with $L_{B}: x-3^{1 / 2} y=0$ for trajectories with final state $B$. (d) Number of crossings with $L_{R}: 3^{1 / 2} x-y=0$ for nonreactive trajectories. Trajectories in the gray peripheral region that do not cross $L_{R}$ at all are reflected by the reactant barrier and never enter the intermediate region. (The dividing lines $L_{A}, L_{B}$, and $L_{R}$ are illustrated in Fig. 7).

Fig. 6 shows, trajectories that reach the same channel from different islands are qualitatively different. In the example of Fig. 6(a), trajectory 2 differs from trajectory 1 by an additional oscillation in the $x$ direction. This difference can be detected by counting the crossings of the trajectory with the line $x=0$ (where we count only crossings from positive to negative $x$ ). As can be seen in Fig. 6(b), trajectories within the same island have the same number of crossings with the dividing line. However, the number of crossings does not uniquely identify the island and therefore does not provide an exhaustive characterization of the island structure. A similar classification can be carried out for trajectories that react into channel $\mathrm{B}$ or return into the reactant channel, although different dividing lines have to be chosen (see Fig. 7). It is shown in Figs. 6(c) and 6(d). In the latter case, there is a large peripheral region of trajectories that do not cross the dividing line at all. These trajectories are reflected by the barrier $S_{R}$ before they can enter into the intermediate region.

A more detailed understanding of the island structure in Fig. 5 can be obtained by noting its similarity with the reactive islands observed by De Leon et al. ${ }^{11-13}$ These authors interpreted the reactive islands in a two-dimensional autonomous system as the imprints of invariant "cylindrical manifolds" that separate reactive from nonreactive trajectories and that intertwine in a complicated manner as they follow

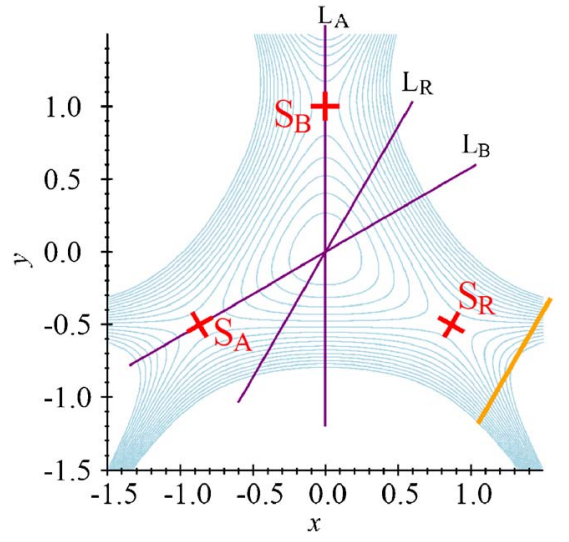

FIG. 7. (Color online) The dividing lines $L_{A}: x=0, L_{B}: x-3^{1 / 2} y=0$, and $L_{R}$ : $3^{1 / 2} x-y=0$ used to characterize the island structure.

the intricacies of a homoclinic tangle. This picture was later generalized to more than two degrees of freedom, ${ }^{1,4,6}$ where the stable and unstable manifolds of the NHIM that were described above play the role of the cylindrical manifolds and show the same complicated behavior. While the theory of Refs. 11-13 can immediately be applied to the timeindependent situation shown in Fig. 5(a), previous approaches are not capable of handling the time-dependent case of Fig. 5(b). However, the time-dependent normal form theory described here enables us to define and compute the corresponding invariant manifolds even for the driven system. We will show that these manifolds determine the structure of reactive islands in the same way as they do in the autonomous setting.

We have constructed the time-dependent normal forms around all three saddle points. The resulting expressions for the NF Hamiltonians and the coordinate transformations are available as supplemental material on EPAPS. ${ }^{35}$ Once a trajectory comes close enough to a barrier for the corresponding NF expansion to be valid, we evaluate the action $\bar{I}_{1}$ of the reactive mode that determines the fate of the trajectory. If $\bar{I}_{1}>0$, the trajectory will cross the barrier, and we assign the value of $\bar{I}_{1}$ as the escape action of that trajectory. If $\bar{I}_{1}<0$, we know that the trajectory will be deflected by the barrier. Thus, trajectories with slightly negative $\bar{I}_{1}$ suffer an entirely different fate from their neighbors with slightly positive $\bar{I}_{1}$ : They return into the intermediate region with its complicated dynamics and later attempt to escape across a different barrier. This sensitivity of trajectories close to the separatrix is the cause of the intricate intertwining of the reactive islands. The separatrices themselves are given by the zeros of the escape action. Trajectories with $\bar{I}_{1}=0$ lie on the stable manifold of the TS trajectory. They are trapped on the barrier and will neither escape nor be reflected.

In practice, we calculate the coefficients in the TDNF expansion for times $t \in[-20.48,+20.48]$. To calculate the $S$ functionals appearing in TDNF, we use fast Fourier transform algorithm ${ }^{36}$ by dividing the interval $t \in[-20.48$, +20.48 ] into $2^{12}$ grid points. For the bath modes, we set $\epsilon=10^{-7}$ in Eq. (29) to calculate the principal value in Eq. (31). The normal form and the action $\bar{I}_{1}$ are evaluated once a 


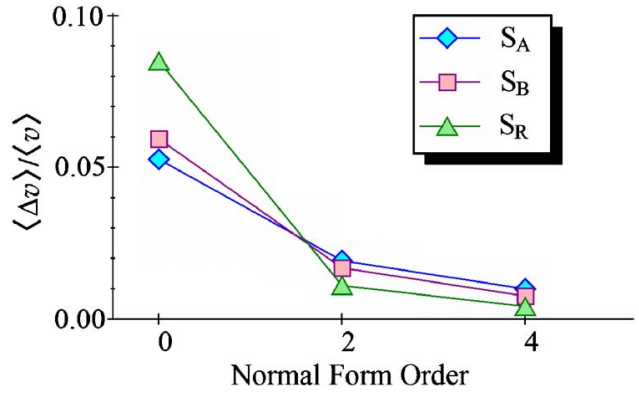

FIG. 8. (Color online) Error in the Hamiltonian vector fields for NF calculation as a function of the NF order. Triangle, diamond, and square symbols show the error for the NF at $S_{R}, S_{A}$, and $S_{B}$, respectively. The decrease of the error means convergence of the polynomical expansions in the NF calculation.

trajectory comes so close to a saddle point that the real normal mode coordinate satisfies $\left|q_{1}^{r}\right|<\eta$, where we choose the threshold $\eta=0.2$ for $S_{R}$ and $\eta=0.12$ for $S_{A}$ and $S_{B}$. The results of the calculation do not depend noticeably on any of these numerical parameters. They do depend on the order $N$ to which the normal form expansion is carried out, and it will be crucial to monitor the convergence of the results with increasing $N$.

The quality of the TDNF expansion can be monitored locally through the error [Eq. (69)] of the Hamiltonian vector field. It is evaluated at the same phase space point and at the same time as the action $\bar{I}_{1}$. To obtain an overall error estimate, we average over all trajectories that lead to escape across a given saddle. We compare the average error $\langle\Delta v\rangle$ to the average of the norm [Eq. (70)] of the Hamiltonian vector field itself, which is evaluated at the same points. Figure 8 shows the relative error $\langle\Delta v\rangle /\langle v\rangle$ for each saddle point and for each TDNF order. The zeroth order corresponds to the harmonic approximation described in Secs. II A and II B. For all the three saddle points, the error decreases as the NF order increases and becomes about $1 \%$ of the norm of the vector field for the fourth order. Thus, we can conclude that the TDNF is a good approximation for this system.

The time-dependent invariant manifolds describe both steps of the reaction, i.e., both the formation and the decay of the activated complex. To study the formation step, we calculate the reactive-mode action $\bar{I}_{1}$ of trajectories that approach the reactant barrier from the reactant side. For our sample of trajectories, the results are shown in Fig. 9. There is in the space of initial conditions an island of positive action where trajectories enter the intermediate region. It is surrounded by a region of negative action whose trajectories are repelled by the barrier. The line $\bar{I}_{1}=0$ forms the separatrix between entering and nonentering trajectories. In all cases, the numerical simulations confirm that the value of the action predicts the fate of a trajectory correctly.

A much more complicated picture is obtained on escape, after the trajectories have taken part in the complex internal dynamics of the activated complex. As described above, we assign a final channel and an escape action to a trajectory once it approaches one of the barriers with a positive action in the reactive mode. The actions obtained in this way are shown in Fig. 10. They take positive values within the reac-

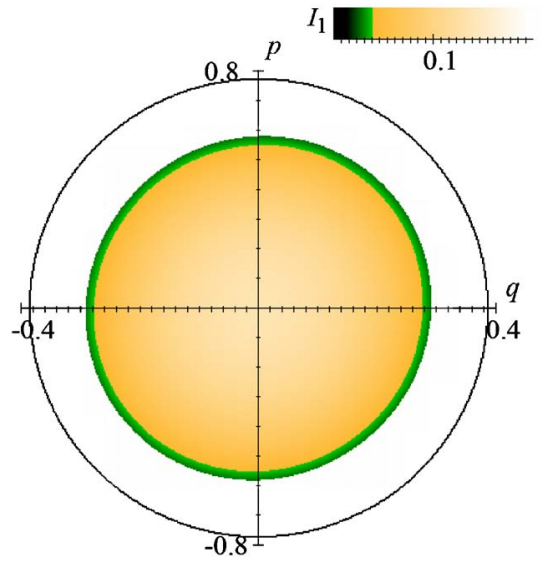

FIG. 9. (Color online) Reactive-mode action $\bar{I}_{1}$ of trajectories first approaching the reactant barrier $S_{R}$. Trajectories with $\bar{I}_{1}>0$ will enter into the intermediate region, those with $\bar{I}_{1}<0$ are reflected. The separatrix between the entering and nonentering trajectories is given by $\bar{I}_{1}=0$. The outer solid curve indicates the boundary of possible initial conditions at energy $E_{0}=0.3$. Trajectories within this region for which no action is given (white) do not come close enough to the saddle for the normal form expansion to be valid.

tive islands and decrease to zero as the border of an island is approached. Since the invariant manifolds of the TS trajectories on the saddles are given by $\bar{I}_{1}=0$, this observation confirms that the borders of the reactive island are formed by these stable manifolds.

In order to assess the validity of the normal-form predictions more accurately, we focus on one of the major reactive islands. Figure 11 shows the boundary of the island around $(q, p)=(-0.22,0)$ that was obtained numerically and compares it to the results of normal form expansions of various orders. The normal-form results converge toward the exact island boundaries with increasing $N$ and practically coincide with them for $N=4$. This confirms the validity of the normal form expansion. Nevertheless, the harmonic approximation differs significantly from the correct results, which shows

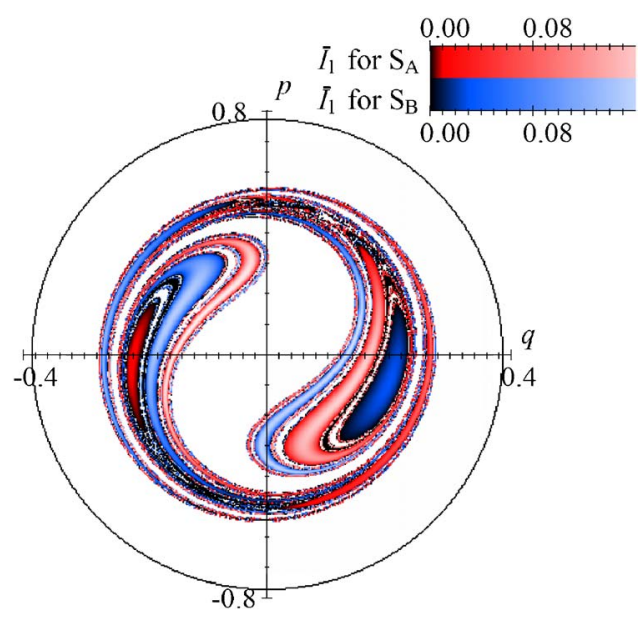

FIG. 10. (Color online) Escape actions as a function of initial conditions, computed from a fourth-order NF expansion. Red, trajectories escaping into channel A; blue, trajectories escaping into B. The outer solid curve indicates the boundary of possible initial conditions at energy $E_{0}=0.3$. In order not to overload the figure, we do not indicate the escape actions of returning trajectories. The actions are positive in the interior of a reactive island and decrease to zero as the border of the island is approached. 


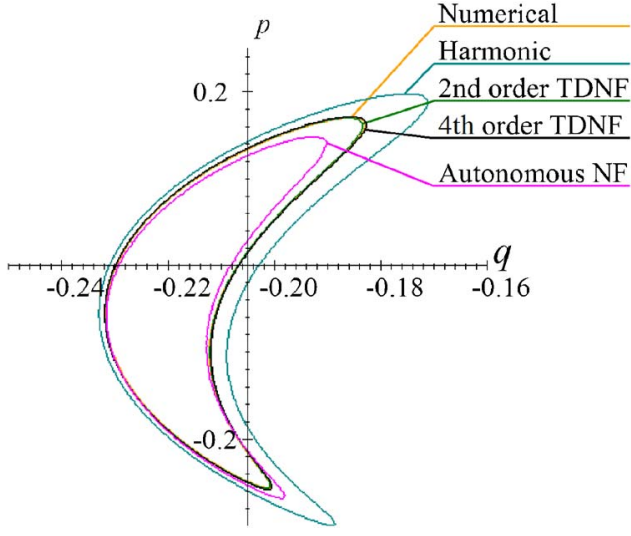

FIG. 11. (Color online) Border of the reactive island around $(-0.22,0)$ obtained numerically and through normal form theory. The results of timedependent NF theory converge toward the exact result with increasing expansion order. Autonomous NF theory (carried out in fourth order) fails.

that the nonlinearities that are incorporated through the higher orders of the expansion have a strong influence on the dynamics.

In addition, Fig. 11 shows the boundary of the island that was obtained from a fourth-order autonomous normal form expansion that neglects the time-dependent driving (in the trajectory calculations, the time dependence was retained). Although this expansion order is large enough to take account of the nonlinearities, the boundary found in this calculation is wrong. Thus, the external driving has a noticeable impact on the escape dynamics, and it can only be described correctly if both the time dependence and the nonlinearities are taken into account. The manifolds calculated here are beyond the reach of earlier approaches that can only handle either the nonlinearities or the time dependence.

A further observation can be adduced to support the identification of the island boundaries with time-dependent invariant manifolds: If this interpretation is correct, the boundaries correspond to trajectories that get trapped on a barrier top for all time. Thus, neighboring trajectories that are close to a boundary should take a long time before they escape. To verify this prediction, we study the section $p=0$ through the island shown in Fig. 11 and calculate the time at which a trajectory crosses the TS dividing surface defined by Eq. (68). Figure 12 shows the escape time as well as the escape action as a function of the initial coordinate $q$. The plot confirms, once again, that the boundary of the island is given by the zero of the reactive-mode action and that trajectories that reach the barrier with negative action do not escape. It also shows, as anticipated, that the escape time grows to infinity as the boundary is approached. We can therefore conclude that the boundaries of the reactive islands are indeed formed by invariant manifolds, just as they are in autonomous systems, and that time-dependent NF theory provides an effective way of calculating them.

\section{CONCLUSION AND OUTLOOK}

In summary, we have developed time-dependent transition state theory as a tool to investigate the dynamics of reactive systems under the influence of external driving. We

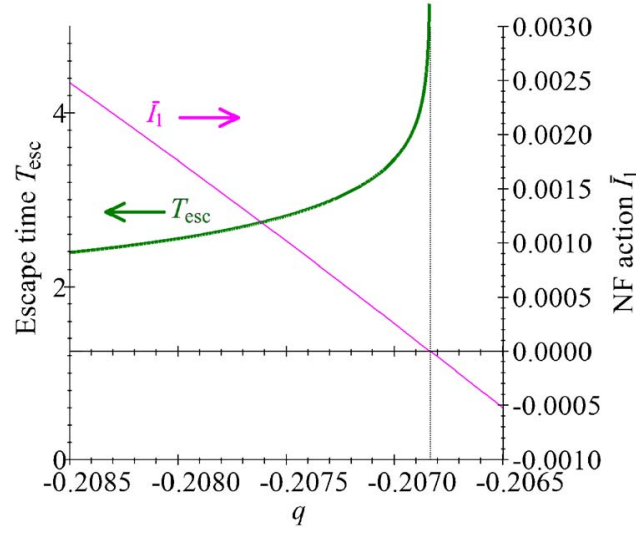

FIG. 12. (Color online) Escape time $T_{\text {esc }}$ and escape action $\bar{I}_{1}$ for trajectories starting on the section $p=0$ through the island shown in Fig. 11. At the border of the island, shown by the dotted line, the action tends to zero, the escape time diverges.

showed how to define a TS trajectory as a generalization of the saddle point in an autonomous system. Time-dependent normal form theory then allows us to incorporate nonlinearities. While resonances between the internal dynamics and the external driving prevent the reduction to an integrable normal form, it is possible to define a reaction coordinate with regular dynamics that separates from the other modes. The action variable associated with the reactive mode defines time-dependent invariant manifolds that act as separatrices between reactive and nonreactive trajectories. In a space of initial conditions, they give rise to reactive islands that are entirely analogous to those found in autonomous systems. Once these islands are known, the ultimate fate of a trajectory can be predicted without a numerical simulation.

We demonstrated the efficacy of this computational scheme by calculating the separatrices for a Hénon-Heiles system with a dipole interaction, which serves as a simple model of a reactive system with several open channels. In this system, we find an intricate pattern of reactive islands similar to that known from time-independent systems. This pattern is accurately described and explained by the invariant manifolds constructed through time-dependent normal form theory. Although we discussed an example system with only two degrees of freedom and dipole interaction, our method can readily be applied to higher-dimensional systems and higher nonlinear laser-molecule interactions.

It will be interesting and rewarding to study the effect of field parameters such as the frequency, pulse duration, and carrier-envelope phase on these separatrices. Such a study will lead to valuable physical insight into why certain pulses enhance a specific reaction and others do not. This may constitute a first step towards controlling chemical reactions by manipulating the location of the separatrices through tailored laser fields.

\section{ACKNOWLEDGMENTS}

This research was supported by the U.S. National Science Foundation and Project No. MTM2005-08595 of Ministerio de Educación y Ciencia (Spain). The authors thank 
Professor Patricia Yanguas in Universidad Pública de Navarra for useful and valuable discussions.

${ }^{1}$ T. Uzer, C. Jaffé, J. Palacián, P. Yanguas, and S. Wiggins, Nonlinearity 15, 957 (2002).

${ }^{2}$ C. Jaffé, S. Kawai, J. Palacián, P. Yanguas, and T. Uzer, Adv. Chem. Phys. 130, 171 (2005).

${ }^{3}$ M. Toda, T. Komatsuzaki, T. Konishi, R. S. Berry, and S. A. Rice, Adv. Chem. Phys. 130A/130B (2005).

${ }^{4}$ H. Waalkens, A. Burbanks, and S. Wiggins, J. Phys. A 37, L257 (2004).

${ }^{5}$ H. Waalkens, A. Burbanks, and S. Wiggins, J. Chem. Phys. 121, 6207 (2004).

${ }^{6}$ F. Gabern, W. S. Koon, J. E. Marsden, and S. D. Ross, Physica D 211 391 (2005).

${ }^{7}$ C.-B. Li, Y. Matsunaga, M. Toda, and T. Komatsuzaki, J. Chem. Phys. 123, 184301 (2005)

${ }^{8}$ C.-B. Li, A. Shojiguchi, M. Toda, and T. Komatsuzaki, Few-Body Syst. 38, 173 (2006).

${ }^{9}$ C.-B. Li, A. Shojiguchi, M. Toda, and T. Komatsuzaki, Phys. Rev. Lett. 97, 028302 (2006).

${ }^{10} \mathrm{~S}$. Wiggins, Normally Hyperbolic Invariant Manifolds in Dynamical Systems (Springer-Verlag, New York, 1991).

${ }^{11}$ A. M. Ozorio de Almeida, N. De Leon, M. A. Mehta, and C. C. Marston, Physica D 46, 265 (1990).

${ }^{12}$ N. De Leon, M. A. Mehta, and R. Q. Topper, J. Chem. Phys. 94, 8310 (1991).

${ }^{13}$ N. De Leon, M. A. Mehta, and R. Q. Topper, J. Chem. Phys. 94, 8329 (1991).

${ }^{14}$ Molecules in Laser Fields, edited by A. D. Bandrauk (Dekker, New York, 1994).

${ }^{15}$ S. A. Rice and M. Zhao, Optical Control of Molecular Dynamics (Wiley,
New York, 2000)

${ }^{16}$ T. Brabec and F. Krausz, Rev. Mod. Phys. 72, 545 (2000).

${ }^{17}$ B. J. Sussman, D. Townsend, M. Y. Ivanov, and A. Stolow, Science 314, 278 (2006).

${ }^{18}$ M. Shapiro and P. Brumer, Phys. Rep. 425, 195 (2006).

${ }^{19}$ T. Bartsch, R. Hernandez, and T. Uzer, Phys. Rev. Lett. 95, 058301 (2005).

${ }^{20}$ T. Bartsch, T. Uzer, and R. Hernandez, J. Chem. Phys. 123, 204102 (2005).

${ }^{21}$ T. Bartsch, T. Uzer, J. M. Moix, and R. Hernandez, J. Chem. Phys. 124, 244310 (2006).

${ }^{22}$ A. Deprit, Celest. Mech. 1, 12 (1969).

${ }^{23}$ E. L. Sibert III, J. Chem. Phys. 88, 4378 (1988).

${ }^{24}$ M. Joyeux and D. Sugny, Can. J. Phys. 80, 1459 (2002).

${ }^{25}$ F. Gabern and À. Jorba, J. Nonlinear Sci. 15, 159 (2005).

${ }^{26}$ M. Hénon and C. Heiles, Astron. J. 69, 73 (1964).

${ }^{27}$ A. J. Dragt and J. M. Finn, J. Math. Phys. 20, 2649 (1979).

${ }^{28}$ R. Hernandez, J. Chem. Phys. 101, 9534 (1994).

${ }^{29}$ À. Jorba, Exp. Math. 8, 155 (1999).

${ }^{30}$ S. Kawai, C. Jaffé, and T. Uzer, J. Phys. B 38, S261 (2005).

${ }^{31}$ A. D. Bandrauk, E.-W. S. Sedik, and C. F. Matta, J. Chem. Phys. 121, 7764 (2004)

${ }^{32}$ S. Chelkowski and A. D. Bandrauk, Phys. Rev. A 65, 061802 (2002).

${ }^{33}$ S. L. Mielke, B. C. Garrett, and K. A. Peterson, J. Chem. Phys. 116, 4142 (2002).

${ }^{34}$ T. H. Dunning Jr., J. Chem. Phys. 66, 2752 (1977).

${ }^{35}$ See EPAPS Document No. E-JCPSA6-126-009715 for the detailed expressions of the TDNF calculation results. This document can be reached via a direct link in the online article's HTML reference section or via the EPAPS homepage (http://www.aip.org/pubservs/epaps.html).

${ }^{36}$ W. H. Press, S. A. Teukolsky, W. T. Vetterling, and B. P. Flannery, Numerical Recipes in C (Cambridge University Press, Cambridge, 1992). 